\title{
Polyethylene Damage Increases With Varus Implant Alignment in Posterior-stabilized and Constrained Condylar Knee Arthroplasty
}

\author{
Zhichang Li MD, Christina I. Esposito PhD, Chelsea N. Koch BS, \\ Yuo-yu Lee MS, Douglas E. Padgett MD, Timothy M. Wright PhD
}

Received: 27 March 2017/Accepted: 10 August 2017/Published online: 18 August 2017

(C) The Association of Bone and Joint Surgeons (B) 2017

\begin{abstract}
Background Implant malalignment in primary TKA has been reported to increase stresses placed on the bearing surfaces of implant components. We used a longitudinally maintained registry coupled with an implant retrieval program to consider whether preoperative, postoperative, or prerevision malalignment was associated with increased risk of revision surgery after TKA.
\end{abstract}

One of the authors (ZL) received funding from the Beijing Joint Care Foundation.

The institution of one or more of the authors (DEP, TMW) has received, during the study period, funding from the Mary and Fred Trump Institute for Implant Analysis.

One of the authors certifies that he (TMW) or a member of his immediate family, has or may receive payments or benefits, during the study period, an amount of USD 100,001-USD 1,000,000 from Exactech Inc (Gainesville, FL, USA).

All ICMJE Conflict of Interest Forms for authors and Clinical Orthopaedics and Related Research ${ }^{\circledR}$ editors and board members are on file with the publication and can be viewed on request.

Clinical Orthopaedics and Related Research ${ }^{\circledR}$ neither advocates nor endorses the use of any treatment, drug, or device. Readers are encouraged to always seek additional information, including FDAapproval status, of any drug or device prior to clinical use.

Each author certifies that his or her institution approved the human protocol for this investigation and that all investigations were conducted in conformity with ethical principles of research.

This work was performed at the Hospital for Special Surgery (New York, NY, USA).

Z. Li, C. I. Esposito, C. N. Koch, T. M. Wright ( $ه)$

Department of Biomechanics, Hospital for Special Surgery, 535

East 70th Street, New York, NY 10021, USA

e-mail:wrightt@hss.edu

\section{Z. Li}

Arthritis Clinic and Research Center, Peking University People's Hospital, Beijing, China
Questions/purposes (1) What is the relative polyethylene damage on medial and lateral compartments of the tibial plateaus from revised TKAs? (2) Does coronal TKA alignment affect implant performance, such that TKAs aligned in varus are predisposed to experience increased polyethylene damage? (3) Does TKA alignment differ between postoperative and prerevision radiographs, and if so, what does this difference suggest about the mechanical contact load placed on a knee with a TKA?

Methods Between 2007 and 2012, we performed 18,065 primary TKAs at our institution. By March 2016, 178 of those TKAs (1\%) were revised at our center at least 2 years after primary surgery at our institution. Eighteen of those TKAs were excluded from this analysis because the tibial insert was not explanted during revision surgery, and four more were excluded because the inserts were lost or returned to the patient before the study was initiated, leaving 156 retrieved polyethylene tibial inserts (in 153 patients) revised at greater than 2 years after the primary TKA for this retrospective study. Patients who underwent revision surgery elsewhere were not considered here, since this study depended on having retrieved components. Polyethylene damage modes of burnishing, pitting, scratching, delamination, surface deformation, abrasion, and third-body debris were subjectively graded on a scale of 0 to 3 to reflect the extent and severity of each damage mode. On preoperative, postoperative, and prerevision

Y. Lee

Department of Epidemiology and Biostatistics, Hospital for Special Surgery, New York, NY, USA

D. E. Padgett

Adult Reconstruction and Joint Replacement Division, Department of Orthopaedic Surgery, Hospital for Special Surgery, New York, NY, USA 
radiographs, overall alignment, femoral alignment, and tibial alignment in the coronal plane were measured according to the protocol recommended by the Knee Society.

Results Knees with more overall varus alignment after TKA had increased total damage on the retrieved tibial inserts (Spearman's rank correlation coefficients of -0.3 [95\% CI, -0.4 to $-0.1 ; p=0.001]$ ). We also found revised TKAs tended to drift back into greater varus before revision surgery, with a mean (SD) of $3.6^{\circ} \pm 4.0^{\circ}$ valgus for postoperative alignment compared with $1.7^{\circ} \pm 6.4^{\circ}$ prerevision $(\mathrm{p}=0.04)$.

Conclusions Despite surgical efforts to achieve neutral mechanical alignment, remaining varus alignment places an increased contact load on the polyethylene articular surfaces. The drift toward further varus alignment postoperatively is consistent with the knee adduction moment remaining high after surgery.

Clinical Relevance While we found a predisposition toward recurrence of the preoperative varus deformity, we did not find increased medial as opposed to lateral polyethylene damage, which may be explained by the curve-oncurve toroidal design of the articulating surfaces of the TKA implants in this study.

\section{Introduction}

While the overall percentage of patients requiring revision TKA is low, more than 700,000 primary TKAs are performed annually in the United States alone [7], resulting in a large number of revision procedures; this carries considerable healthcare and financial implications. Schroer et al. [28] reported on the reasons for revision of more than 800 TKAs during 2010 and 2011 at six arthroplasty centers in the United States; approximately half were related to malalignment of the TKA components and wear of the polyethylene tibial insert. Malalignment before and after primary TKA have been linked to decreased implant survival [27] and inferior patient-reported outcomes [17]. Conversely, proper component alignment is strongly associated with greater stability, a lower likelihood of loosening, and less polyethylene wear [5, 13, 26], although not all studies have concurred on this finding [6, 19, 23].

Implant malalignment in primary TKA has been reported to increase the stresses placed on the bearing surfaces and the fixation interfaces between the implant components and the surrounding cement and bone [9, 10]. This has been supported by implant retrieval analysis studies of cruciate retaining (CR) [30, 32] and posteriorstabilized (PS) implants in TKAs [22], with both types of implants showing increased polyethylene damage with implant malalignment. However, major limitations of these studies include the reliance on prerevision rather than postoperative radiographs to measure alignment, the absence of preoperative radiographs before the index arthroplasty to see whether the initial deformity has a propensity to recur, the focus on a single CR or PS design, and the inclusion of results from primary and revision TKAs. A large, longitudinally maintained registry coupled with an implant retrieval program could be used to consider the effect of implant alignment on polyethylene damage while controlling for patient variables (such as age and BMI) and implant characteristics (like the design and level of constraint), a marked improvement over previous studies [22, 30, 32]. Having serial radiographs from which to measure preoperative, postoperative, and prerevision alignment would provide additional valuable information to assess how surgical alignment affects TKA outcome.

We therefore combined our institutional registry of TKA radiographs and our implant-retrieval program to ask three research questions: (1) What is the relative polyethylene damage on medial and lateral compartments of the tibial plateaus from revised TKAs? (2) Does coronal TKA alignment affect implant damage, such that TKAs aligned in varus are predisposed to experience increased polyethylene damage? (3) Does TKA alignment differ between postoperative and prerevision radiographs, and if so what does this difference suggest about the mechanical contact load on a knee with a TKA?

\section{Patients and Methods}

From May 2007 to December 2012, 18,065 primary TKAs performed in 16,083 patients at a single institution were recorded in an institutional review board-approved longitudinally maintained total joint arthroplasty registry. Patient demographics, preoperative health status, medical comorbidities, and clinical and patient-reported outcome measures were collected. If patients later underwent revision surgery at our institution, the date and reason for revision surgery were recorded as a part of the registry.

By March 2016, 417 (2\%) of the primary TKAs in the registry had been revised (Fig. 1). All patients had their index and revision operations performed at our institution. We did not account for patients who had revisions performed at outside institutions. For our study, 239 TKAs that underwent revision less than 2 years after surgery were excluded because a minimum of 2 years followup typically is required for publishing clinical outcomes after joint arthroplasty, and because these patients may not have fully returned to their normal activities of daily living, and therefore the damage patterns on their retrieved polyethylene tibial inserts may not reflect implant alignment. Of the remaining 178 primary TKAs, another 18 were excluded because the tibial insert was not explanted during 
revision surgery, and four more were excluded because the inserts were lost or returned to the patients before the study was started. Therefore, we report on 156 retrieved polyethylene tibial inserts (in 153 patients) revised at greater than 2 years after the primary TKA.

The patients ( 46 males, 107 females) had a mean age of 61 years (range, 28-86 years) and a mean BMI of $31 \mathrm{~kg} / \mathrm{m}^{2}$ (range, $18-51 \mathrm{~kg} / \mathrm{m}^{2}$ ). Reasons for revision surgery included aseptic loosening in 51 TKAs (33\%), instability in $46(29 \%)$, infection in $25(16 \%)$, stiffness in $12(8 \%)$, periprosthetic fracture in seven $(4 \%)$, and other reasons, such as persistent pain, in $15(10 \%)$. Sixty-three $(41 \%)$ of the implants were manufactured by Exactech (Gainesville, FL, USA), 50 (32\%) by Smith \& Nephew (Memphis, TN, USA), 30 (19\%) by Zimmer (Warsaw, IN, USA), and 13 $(8 \%)$ by other manufacturers. Most of the implants were PS designs (122 or $78 \%$ ), while the remaining 34 (or 22\%) were constrained condylar knees (CCK).

\section{Visual Assessment of Damage}

Polyethylene damage modes of burnishing, pitting, scratching, delamination, surface deformation, abrasion, and third-body debris were subjectively graded on a scale of 0 to 3 to reflect the extent and severity of each damage mode [15]. Two observers (ZL, CNK) who were blinded to the reasons for revision surgery independently assessed damage on the articular surfaces in each region using a light stereomicroscope $(\times 10$ to $\times 32$ magnification $)$. To assess regional differences in damage to the retrieved tibial inserts, the medial and lateral compartments were divided into four regions (Fig. 2A). Because our research questions

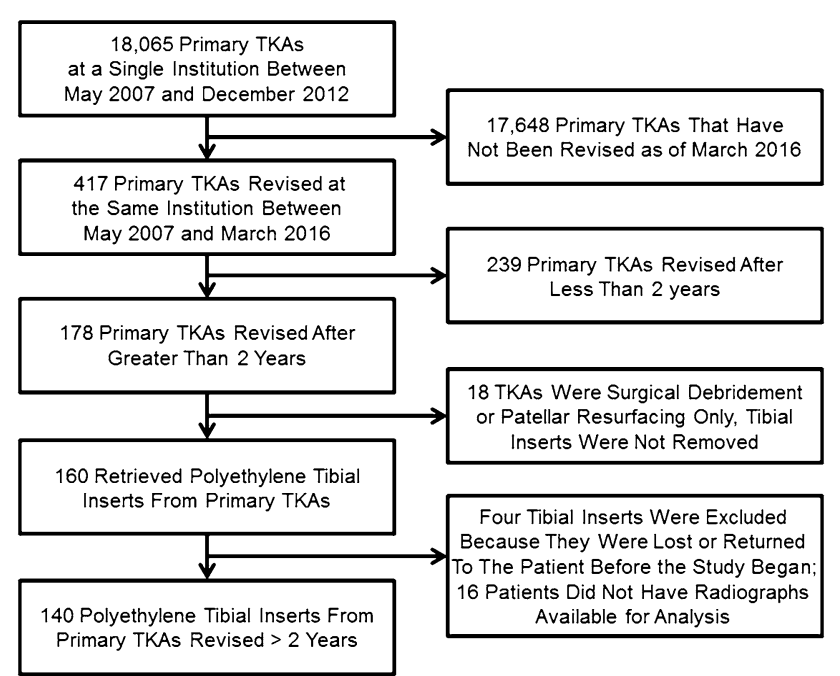

Fig. 1 A flow diagram shows the method for selecting retrieved TKA implants in this study. dealt with the effect of coronal alignment on polyethylene damage, only the medial and lateral surfaces (not the anterior or posterior surfaces) of the PS and CCK tibial posts were graded. The backsides of the tibial inserts also were divided into four regions (Fig. 2B). If the difference in total damage scores summed across all regions was greater than 10 points between the two observers, then a third observer (EB) scored the insert as well. The interobserver kappa statistics for the damage grading was 0.80 , which is considered good agreement for interobserver variability [1]. The maximum total damage score for each of the 13 regions on the tibial insert was 21 (seven damage modes $\times$ maximum score of 3 for each mode). Therefore, the medial and lateral compartments and the backside had maximum possible scores of 84 (four regions $\times$ seven damage modes $\times$ maximum score of 3 for each mode). The posts had a maximum possible score of 42 (two regions $\times$ seven damage modes $\times$ maximum score of 3 for each mode).

\section{Radiographic Analysis}

Preoperative knee alignment, postoperative TKA alignment (at approximately 6 weeks after TKA), and prerevision TKA alignment were measured on weightbearing AP-view radiographs. For our first research question, we had 151 postoperative radiographs of TKAs available (from the 156 retrieved TKA implants) to evaluate the effect of postoperative alignment on implant damage. For our second research question, radiographs were available at all three times for 140 of the 156 TKAs (Fig. 1) in our institutional picture archiving and communication system (PACS, Sectra Imtec AB, Linköping, Sweden). The radiographs from these 140 TKAs were used to evaluate alignment differences between preoperative, postoperative, and prerevision radiographs.

The overall anatomic knee alignment was defined as the angle between the femoral anatomic axis and tibial

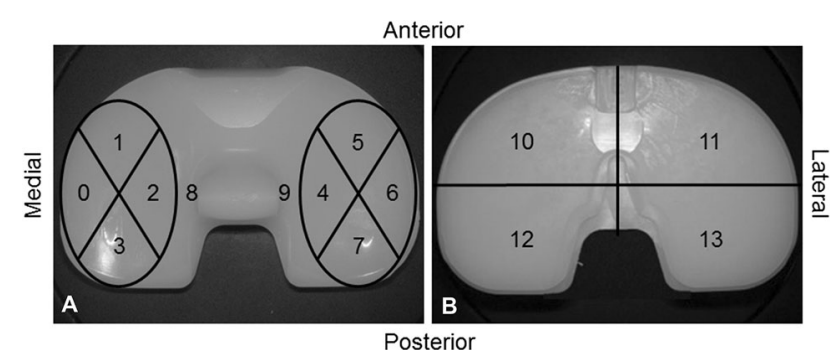

Fig. 2A-B All TKAs in this study used posterior-stabilized implants. The (A) top surface of the polyethylene tibial insert was divided in 10 regions and the (B) backside surface was divided in four zones for damage scoring. 
anatomic axis and was expressed as a deviation from $180^{\circ}$ with varus being a negative and valgus being a positive value (Fig. 3) [24]. Anatomic axes of the femur and tibia were defined as a line drawn through two mid-points between the outer boundaries of the bones at distances of $100 \mathrm{~mm}$ and $150 \mathrm{~mm}$ from the joint line [29].

The alignments of the femoral and tibial components in the coronal plane were measured according to the protocol recommended by the Knee Society [18]. Femoral component alignment was defined as the angle between the distal surface of the femoral component and the anatomic axis of the femur and was expressed as a deviation from $90^{\circ}$ with varus being a negative and valgus being a positive value. Tibial component alignment was defined as the angle between the inferior surface of the tibial baseplate and the anatomic axis of the tibia, with greater than $90^{\circ}$ for a valgus component and less than $90^{\circ}$ for a varus component. All alignment measurements were performed using the PACS system, with an accuracy of $1 \mathrm{~mm}$ for distance and $1^{\circ}$ for angle.

All radiographs were reviewed by two observers (ZL, CIE), who had no preevaluation knowledge of the patients' demographic or clinical data. The interobserver reliability for the radiographic examinations was estimated using intraclass correlation coefficients (ICCs). The interobserver kappa statistics for the radiographic alignment measurements ranged from 0.72 to 0.92 , which is considered good to excellent agreement for interobserver variability [1].

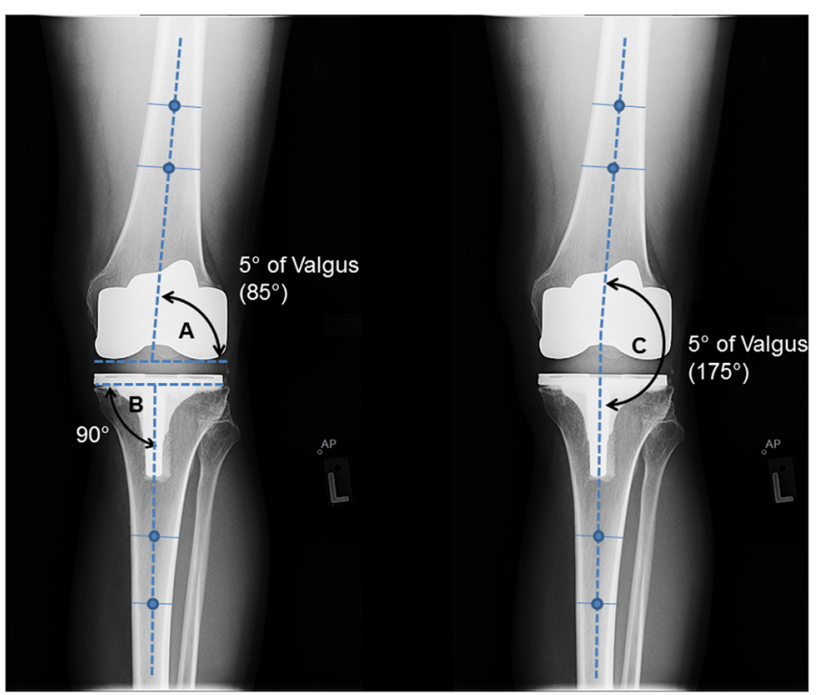

Fig. 3 The same radiograph is shown twice in this figure to show the angles measured for femoral alignment (angle A), tibial alignment (angle B), and overall alignment (angle C) of a TKA. This postoperative AP radiograph is from a 52-year-old woman who had a left TKA, which was revised 3 years later for instability. The femoral component alignment (angle A) was $5^{\circ}$ valgus, the tibial component alignment (angle B) was $90^{\circ}$, and overall anatomic knee alignment (angle $\mathrm{C}$ ) was $5^{\circ}$ valgus.
Statistical Analysis

Damage scores were summarized using mean values with SDs. Damage scores were compared among regions using $t$ tests. Overall knee alignment, femoral component alignment, and tibial component alignment were analyzed as continuous and categorical variables. We used a previously established classification system $[5,14,28]$ to categorize the ranges for varus, neutral, and valgus for overall knee alignment (varus $<3^{\circ}$, neutral $3^{\circ}$ to $7^{\circ}$, valgus $>7^{\circ}$ ) and individual alignment of the tibial (varus $80^{\circ}$ to $86^{\circ}$, neutral $87^{\circ}$ to $93^{\circ}$, valgus $94^{\circ}$ to $95^{\circ}$ ) and femoral components (varus $-3^{\circ}$ to $3^{\circ}$, neutral $4^{\circ}$ to $7^{\circ}$, valgus $8^{\circ}$ to $18^{\circ}$ ). When analyzing alignment as a continuous variable, the relationship between alignment and implant damage was assessed using Spearman's rank correlation coefficient. When analyzing alignment as a categorical variable, differences in implant damage among knees aligned in varus, neutral, and valgus were determined using one-way ANOVA. Subsequently, multiple linear regression analyses were performed to examine the effect of alignment on implant damage, controlling for patient baseline demographics (age, BMI, and sex) and implant characteristics (length of implantation, reason for revision, PS versus CCK, and implant manufacturer). Differences in implant damage based on reason for revision also were examined using ANOVA. Multiple logistic regression analyses were performed to examine the effect of implant damage as affected by the binary outcome variable of reason for revision surgery (aseptic loosening and instability versus all other reasons for revision), while controlling for patient baseline demographics and implant characteristics.

\section{Results}

\section{Medial Versus Lateral Polyethylene Damage Scores}

All 156 retrieved tibial inserts showed damage in the form of pitting (mean score, $20 \pm 9$ ), scratching (mean score, 16 \pm 9 ), and burnishing (mean score, $13 \pm 7$ ). The mean total damage score for all seven damage modes for the top surface of the inserts (zones 0 through 9; Fig. 2A) was 44 \pm 17 and for the backside (zones 10 through 13; Fig. 2B) was $11 \pm 6$. Deformation (94\% of the inserts), abrasion (46\%), embedded debris (19\%), and delamination (8\%) also were present on some of the inserts, but the scores for these damage modes were quite low (mean scores of $4 \pm 3$, $1 \pm 1,0.4 \pm 1,0.4 \pm 1$, respectively).

No difference was found in the damage scores between the medial $(20 \pm 8)$ and lateral $(19 \pm 7)$ compartments of the inserts $(p=0.06)$. Similarly, no differences were found between the medial and lateral regions of the backside of 
the tibial inserts (zones 10 and 12 versus 11 and 13; mean score for both regions, $5 \pm 3 ; \mathrm{p}=0.48$ ). However, more damage was found on the medial region of the tibial post (zone 8; mean damage score, $2.8 \pm 2.3$; 95\% CI, 2.443.18) compared with the lateral region of the tibial post (zone 9; mean damage score, $2.6 \pm 2.0$; 95\% CI, 2.262.88), with a mean difference of 0.24 (95\% CI, 0.06-0.42; $\mathrm{p}=0.008)$.

\section{Postoperative TKA Alignment and Implant Damage}

Knees with more overall varus alignment after TKA had increased total damage on the retrieved tibial inserts (Spearman's rank correlation coefficients of -0.3 [95\% CI, -0.4 to $-0.1 ; \mathrm{p}=0.001]$ ), as did those with varus femoral component alignment (Spearman's rank correlation coefficients of -0.2 [95\% CI, -0.4 to $-0.06 ; \mathrm{p}=0.009$ ]) and varus tibial component alignment (Spearman's rank correlation coefficients of -0.2 [95\% CI, -0.06 to $-0.4 ; \mathrm{p}=$ $0.017]$ ). When defining overall knee alignment as a categorical variable (varus, neutral, or valgus), we found an increase in implant damage in all regions of the insert when a TKA had overall varus alignment (Fig. 4). Interestingly, the least damage on the polyethylene inserts occurred in TKAs with overall valgus alignment (mean total damage score $63 \pm 23$ for varus versus $45 \pm 15$ for valgus; $\mathrm{p}<$ 0.01) (Table 1). We evaluated damage scores for knees revised for reasons other than loosening, knees with preoperative varus alignment, or knees with preoperative valgus alignment to consider the impact of preoperative alignment or risk of loosening on damage (Table 1). Significant differences were only found when postoperative knee alignment for all knees, including those revised for loosening, were evaluated for damage.

After controlling for differences in patient baseline demographics and implant characteristics we found that varus TKA alignment was associated with increased total implant damage (1.2 point increase in total damage for every $1^{\circ}$ increase in varus alignment; $\left.\mathrm{p}<0.01\right)$, lateral damage $\left(0.3\right.$ point increase in total damage for every $1^{\circ}$ increase in varus alignment; $p=0.02)$, post damage $(0.2$ point increase in total damage for every $1^{\circ}$ increase in varus alignment; $\mathrm{p}<0.01)$, and backside damage ( 0.4 point increase in total damage for every $1^{\circ}$ increase in varus alignment; $\mathrm{p}<0.01$ ) (Table 2). Total damage on the insert was greater for CCK as opposed to PS designs (15.6 point increase in total damage for CCK implants compared with PS implants; $\mathrm{p}<0.01)$, and with decreased BMI ( 0.4 point increase in total damage for every 1 unit increase in BMI; $\mathrm{p}=0.03$ ). Total damage scores on the articulating surface were higher in implants removed for loosening compared with those removed for stiffness (regression coefficient

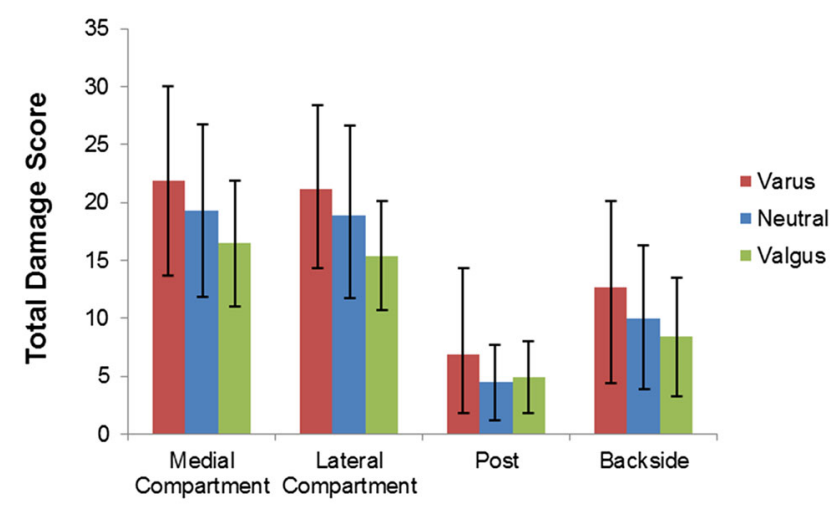

\section{Regions of the Tibial Insert}

Fig. 4 The bar chart shows the damage scores on different regions of the tibial insert. The TKAs were divided into those that were placed with varus (red), neutral (blue), or valgus (green) postoperative alignment. Implants placed with postoperative varus alignment had higher mean damage scores on the medial compartment (21.9 for varus, 19.3 for neutral, and 16.5 for valgus; $p=0.01$ ), lateral compartment (21.2 for varus, 18.9 for neutral, and 15.4 for valgus; $\mathrm{p}<0.01$ ), post (6.9 for varus, 4.5 for neutral, and 4.9 for valgus; $\mathrm{p}<$ 0.01 ), and backside surfaces (12.7 for varus, 10.0 for neutral, and 8.4 for valgus; $p=0.01$ ) of the polyethylene. Maximum damage score is 84 for the medial compartment, lateral compartment, and backside, while the maximum score is 42 for the post.

-33.6 stiffness versus loosening; $\mathrm{p}<0.01)$ or infection (regression coefficient -21.8 infection versus loosening; $\mathrm{p}<0.01$ ). Comparisons to other reasons for revision (Table 2) and the mean total damage scores for different reasons for revision surgery are shown (Fig. 5).

\section{Variability in Alignment With Time}

Preoperative knee deformity tended to be corrected to neutral postoperatively and then recur before revision surgery, with the average measurements (mean $\pm \mathrm{SD}$ ) for preoperative, postoperative, and prerevision overall alignment being valgus $2.5^{\circ} \pm 8.4^{\circ}$, valgus $3.6^{\circ} \pm 4.0^{\circ}$, and valgus $1.7^{\circ} \pm 6.4^{\circ}$, respectively $(\mathrm{p}=0.04)$. Similarly, differences also were found among preoperative, postoperative, and prerevision femoral alignment (valgus $8.6^{\circ} \pm$ $2.8^{\circ}$, valgus $5.2^{\circ} \pm 3.0^{\circ}$, and valgus $4.4^{\circ} \pm 4.5^{\circ}$, respectively; $\mathrm{p}<0.01$ ) and tibial alignment (valgus $3.9^{\circ} \pm 3.7^{\circ}$, valgus $1.3^{\circ} \pm 2.4^{\circ}$, and valgus $2.4^{\circ} \pm 3.6^{\circ}$, respectively; $\mathrm{p}<0.01)$. As expected, postoperative TKA alignment showed less variability (range, $9^{\circ}$ varus to $16^{\circ}$ valgus) and more correction toward neutral compared with preoperative TKA alignment (range, $20^{\circ}$ varus to $26^{\circ}$ valgus). However, by the time the patients had experienced complications with their TKA and were facing revision surgery, the prerevision TKA alignment (Fig. 6) had an average of $2^{\circ}$ more varus compared with postoperative alignment 


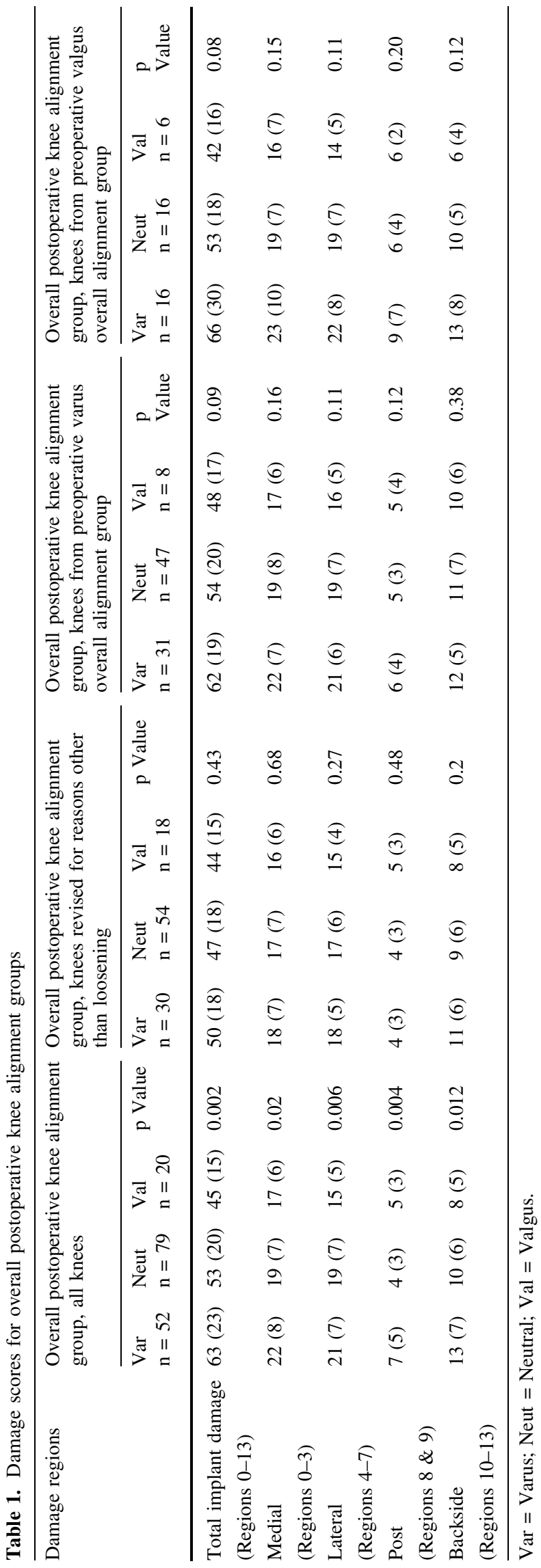

measurements (range, $22^{\circ}$ more varus to $11^{\circ}$ more valgus; $\mathrm{p}<0.01$ ). Therefore, prerevision alignment drifted back toward varus, with an average overall alignment of $1.7^{\circ}$ valgus compared with $3.7^{\circ}$ valgus postoperatively (Fig. 6). Of the 74 patients who had neutral postoperative overall TKA alignment $\left(3^{\circ}\right.$ to $\left.7^{\circ}\right)$ and radiographs at all three times available for analysis, $38(51 \%)$ had greater varus alignment and 29 (39\%) had more valgus alignment before revision surgery (Fig. 7A). The changes in alignment were influenced by the reason for revision surgery of the TKAs. TKAs tended to drift back into varus, with 32 of 45 (71\%) TKAs revised for aseptic loosening, 18 of $45(40 \%)$ revised for instability, four of 12 (33\%) revised for stiffness (33\%), and three of $22(14 \%)$ revised for infection drifting back into varus (Fig. 7B). TKAs with varus postoperative alignment tended to maintain varus alignment, with 41 of the $49(84 \%)$ postoperative varus TKAs having varus alignment before revision surgery. The same was found with valgus TKA alignment $\left(>7^{\circ}\right)$, with 16 of 17 postoperative valgus TKAs (94\%) having valgus alignment before revision surgery.

\section{Discussion}

Although primary TKA has shown its effectiveness by reducing knee pain and increasing knee function in activities of daily living, a general consensus regarding best alignment of the femoral and tibial components is lacking. In this study, which combined the strength of a registry and an implant-retrieval program, we sought to determine whether postoperative overall varus TKA alignment increases polyethylene damage in modern primary PS and CCK implant designs. We found TKAs aligned in postoperative overall varus are predisposed to experience increased polyethylene damage, but the damage was not higher in the medial compartment than the lateral compartment. In addition, we found that revised TKAs tended to drift back into greater varus as shown by comparing postoperative with prerevision radiographs of the same patients (Figs. 6D and 7A), despite preoperative limb alignment or surgical efforts to correct the knee to neutral mechanical alignment.

\section{Limitations}

Inherent limitations of any retrieval study are that such studies are retrospective for which certain variables such as reason for revision cannot be controlled and that the cohort includes implants that by definition have undergone revisions and thus may not reflect well-functioning devices. The patients who returned to our institution may not have 


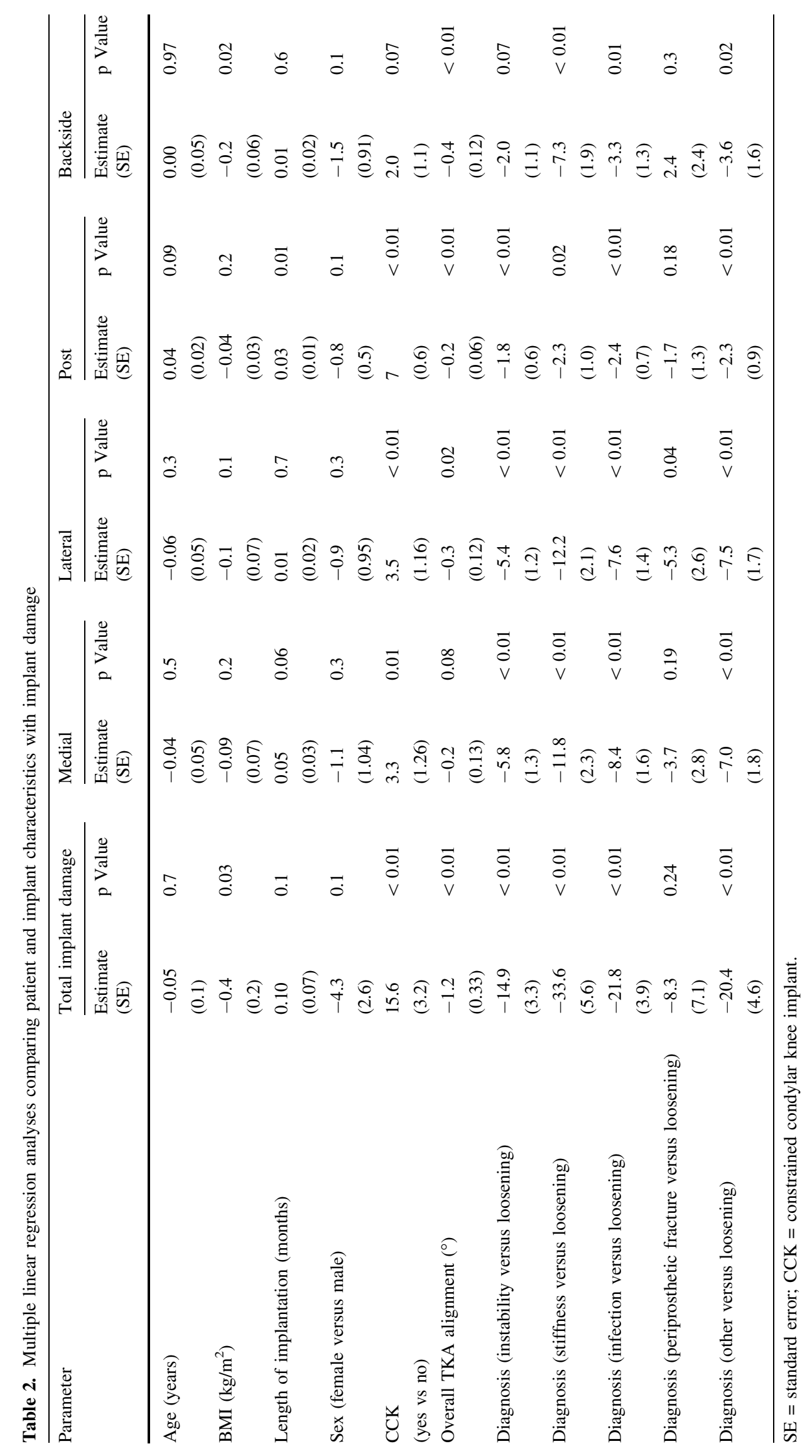


been representative of the overall population who underwent TKAs done during this time, and patients who underwent revision surgery may be more or less likely to return to the original surgeon. However, we sought to address the relationship between revision and tibial insert damage, therefore focusing on implants retrieved at

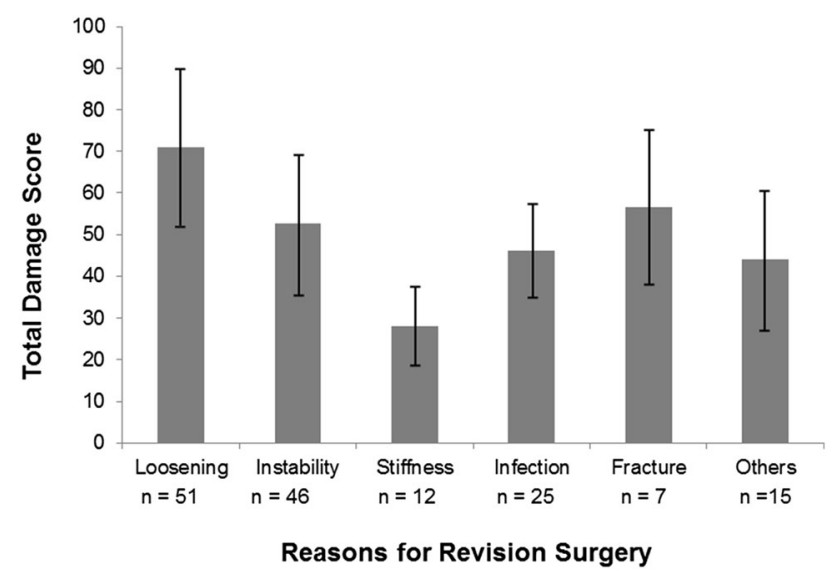

Fig. 5 A bar chart shows the mean total damage scores on the surfaces of tibial inserts revised for different reasons.

25

\section{Preoperative Alignment}

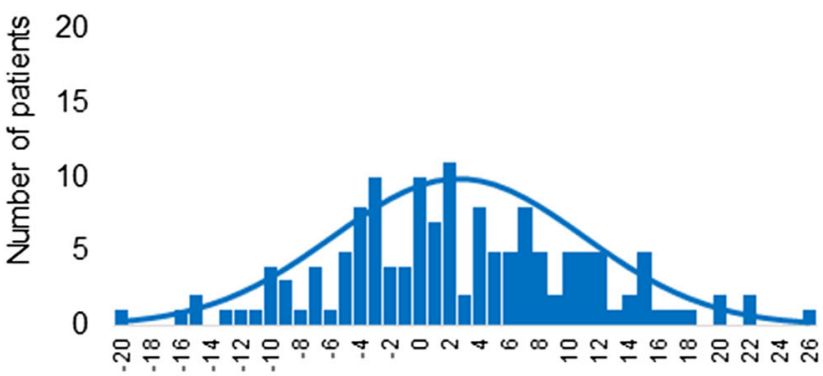

A Varus Overall Knee Alignment $\left({ }^{\circ}\right)$ Valgus

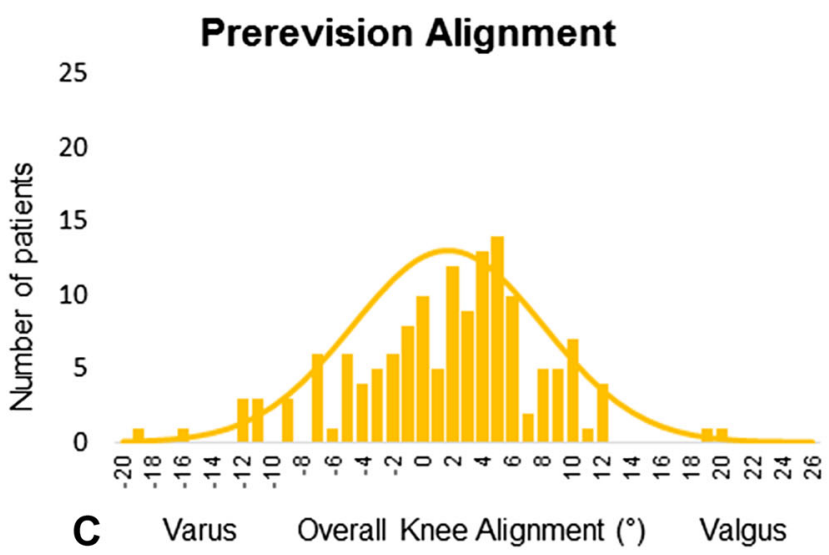

Fig. 6A-D The bar charts show distribution of (A) preoperative, (B) postoperative, and (C) prerevision overall TKA alignment in patients undergoing TKA. (D) The distribution for preoperative, revision surgery was necessary. We did not take into account all the implant design differences among manufacturers that may have contributed to polyethylene damage (including post geometry, tray surface finishes, and polyethylene material properties. However, $92 \%$ of the implants (143 of 156) were manufactured by one of three companies and all were condylar TKA designs. In addition, we used implants retrieved from a large registry, therefore we had nearly complete clinical, demographic, and radiographic information for our patients. Like most studies of alignment after TKA, our study was focused on the static, coronal plane alignment and we were limited to measuring knee alignment on conventional two-dimensional radiographs, since full-length weightbearing radiographs or CT scans are not routinely ordered at our institution owing to the greater cost and increased radiation exposure. Therefore, we did not evaluate sagittal plane or rotational alignment, the combined effects of coronal, sagittal, and rotational alignment, or the dynamic effect of gait and other activities on alignment. Third, we do not know if the damage present on the surface of the implant was related to wear (removal of polyethylene from the surface in the form

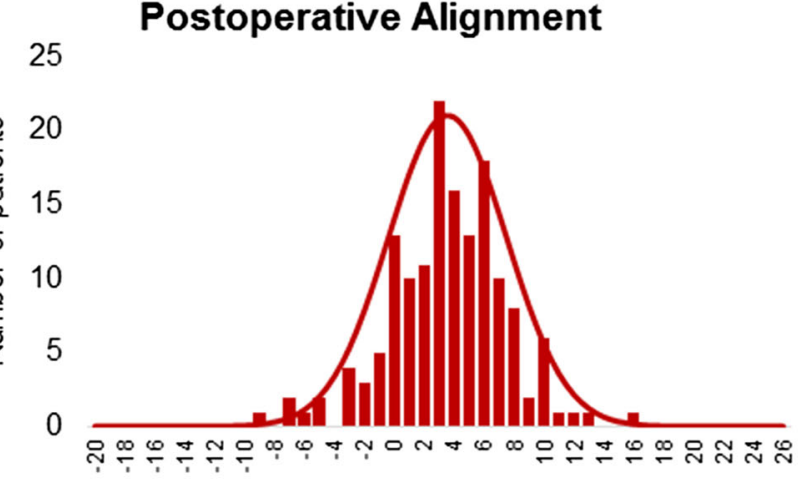

B Varus Overall Knee Alignment $\left({ }^{\circ}\right)$ Valgus

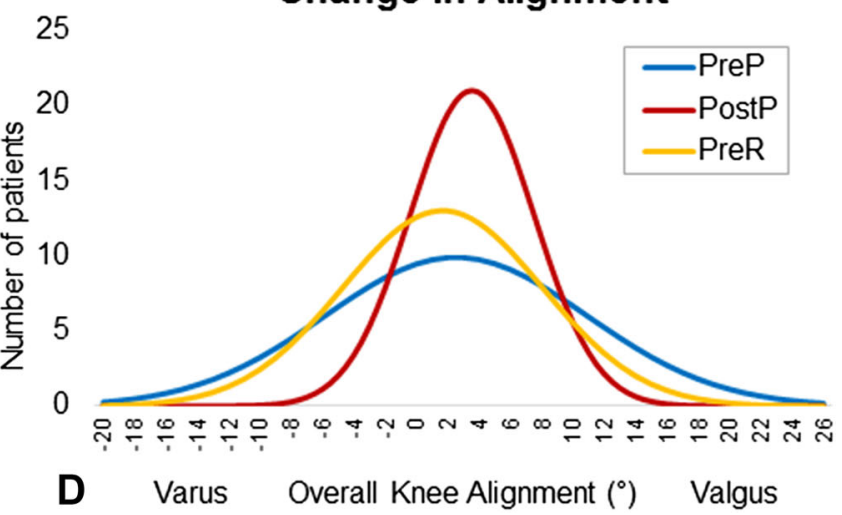

postoperative, and prerevision alignment changed, with a shift back toward varus alignment from postoperative to prerevision times. PreP = Preoperative PostP $=$ Postoperative PreR $=$ Prerevision . 


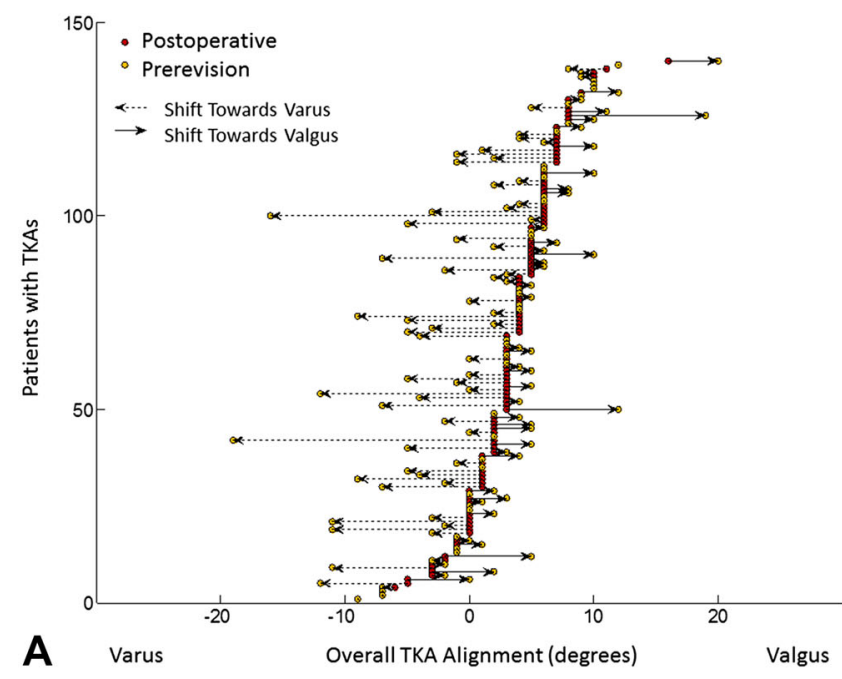

Fig. 7A-B (A) A scatterplot shows the preoperative, postoperative, and prerevision overall TKA alignment for each patient. On the yaxis, each patient is represented at one mark, such that each horizontal line on the graph should have two dots showing the alignment for the patient postoperatively and before revision surgery. The data were sorted according to postoperative TKA alignment, such that the red dots on the graph represent where the TKA was originally aligned.

of debris) or how much of an increase in damage leads to an increase in revision rate. While we have associated damage with malalignment, it remains unclear whether the damage contributed to poor implant survivorship or whether the damage was a byproduct of the contact mechanics caused by malalignment. However, this is an inherent limitation to all retrieval studies of revised implants.

\section{Influence of Alignment on Polyethylene Damage}

We found that varus TKA alignment was associated with increased polyethylene damage, which is supported by prior retrieval studies $[8,22]$. We theorized that this relationship may exist, as Ritter et al. [27] showed coronal TKA alignment as measured on a prerevision AP radiograph reliably predicted the risk of revision surgery, with varus overall alignment associated with collapse under the medial tibial plateau, and valgus overall alignment associated with instability. Given the increased revision rates that they reported of overall tibiofemoral alignment were outside the range of $2.5^{\circ}$ or greater varus and $7.4^{\circ}$ or less valgus, we anticipated that polyethylene damage would be highest with varus or valgus malalignment. Interestingly, we found varus alignment was associated with increased damage, but valgus malalignment (mean damage score, 45) showed the least damage compared with varus or neutral alignment groups (mean damage scores, 63 and 53, respectively; Table 1). Varus tibial component alignment

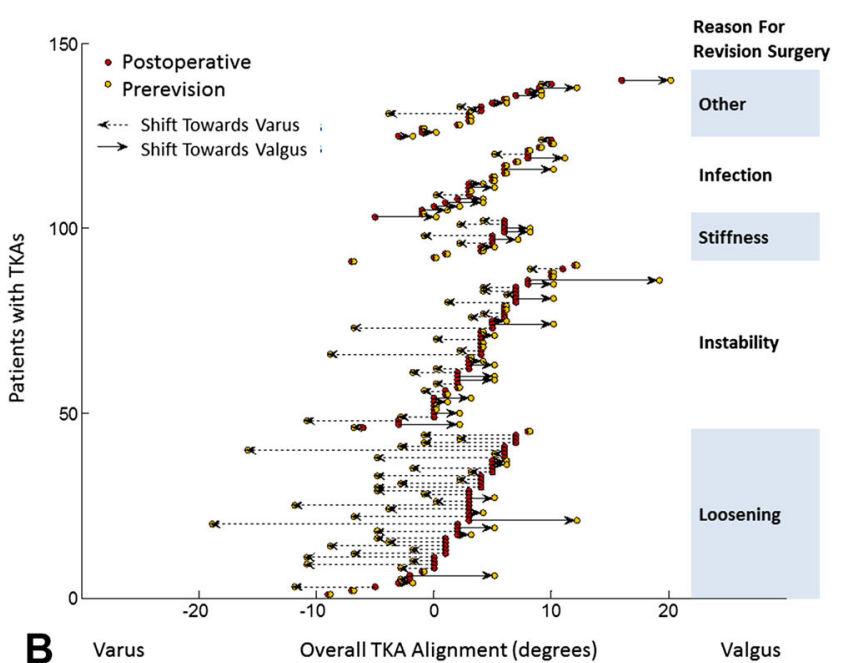

Most prerevision alignment measurements (yellow dots) are to the left of the postoperative measurements (red dots), showing that most TKAs fall into varus. (B) The scatterplot uses the same patients as those used for Illustration A but the data were sorted according to reason for revision surgery. Most TKAs revised for loosening fall into varus, as seen by the prerevision alignment measurements (yellow dots) being to the left of the postoperative measurements (red dots).

may not be detrimental as long as it is paired with a femoral component aligned in valgus. This finding also can be explained by considering the reduction in the adduction moment during gait if the lower limb is in valgus [3]. While overall alignment was found to be associated with increased polyethylene damage, we did not find a substantial increase in polyethylene damage when the tibial component was in varus. Finding a correlation between overall TKA alignment, but not tibial alignment, and higher damage was reported in another retrieval study of 83 PS TKA inserts [22]. While a particular postoperative tibiofemoral knee alignment in the coronal plane may be found to be more advantageous in limiting tibial bearing polyethylene damage (or tibial component loosening), how such a postoperative tibiofemoral knee alignment may affect collateral soft tissue balancing, bone resections, kinematic function of the knee, patellofemoral tracking, and comfort of the patient also must be considered by the operating surgeon. Furthermore, properly balancing the soft tissues about the knee arthroplasty remains important beyond simply reconstructing the knee to a preferred tibiofemoral alignment.

We did not find regional differences in polyethylene damage on the surfaces (for example, increased medial damage in TKAs with excessive varus alignment). This is because TKAs do not solely experience varus and valgus moments during activities of daily living $[4,16]$. Another explanation could be the effect of implant design. For example, Ritter et al. [27] reported on the effect of knee alignment on implant survival by evaluating a large series 
of TKAs of the $\mathrm{AGC}^{\mathbb{R}}$ knee implant design (Biomet, Warsaw, IN, USA). That specific implant design has considerably flatter mediolateral geometries on the condylar surfaces of the femoral components and the articular surfaces on the plateaus of the tibial component compared with the curve-on-curve toroidal articulating surfaces used in the PS and CCK knee designs in our study. Under varus and valgus moments, the flatter design of the $\mathrm{AGC}^{\circledR}$ knee implant could result in edge loading of the femoral component on a relatively small contact area on the outer edges of either the medial or lateral tibial compartments [33]. Although long-term clinical studies have not found edge loading to be a problem with the $\mathrm{AGC}^{\mathrm{R}}$ knee implant $[14,26]$, the geometries of the articular surfaces may influence regionally (medial versus lateral) damage on the plateau and how well a specific implant design may perform when malaligned. A more recent study showed greater damage in the lateral compartment of varus-aligned TKAs and attributed this result to lateral condylar lift-off inducing impact and shear loading in these knees [31]. Our results do not support this finding.

Interestingly, we found increased polyethylene damage on the medial and lateral surfaces of the tibial posts in primary TKAs with CCK implants compared with primary TKAs with PS implants. That the tibial posts, regardless of design, showed damage is evidence that the post was contacting the intercondylar box of the femoral component during in vivo use. This added constraint provides another load transfer pathway in addition to the articular surfaces, and also could partially explain the lack of findings of regional (medial versus lateral) differences. This additional constraint was not present in the $\mathrm{AGC}^{\mathbb{R}}$ cruciate retaining design used by Ritter et al. [27]. The difference between the CCK and PS designs reflects differences in the amount of constraint, with the CCK providing additional constraint through a thickened tibial post with tighter spacing in the intercondylar box [11]. The higher damage on the CCK implants shows that this constraint mechanism was functional, suggesting that the use of a CCK design in some primary TKAs is reasonable [2]; however, a disadvantage of the added constraint may be increased interfacial stresses around the femoral component leading to early loosening [21].

Other factors influenced the damage observed on our retrieved tibial inserts. For example, damage scores were associated with reasons for revision, with TKAs revised for loosening or instability having tibial inserts with more damage than those revised for stiffness or infection (Fig. 5). Patients with stiffness or infection are likely to be less demanding of their implant, and the reduced mechanical burden translates to less surface damage to the polyethylene. In addition, we found increased implant damage associated with increased BMI. Increasing BMI was shown to increase the risk of TKA failure [27], suggesting that poor implant alignment combined with a high BMI represents a greater risk to implant survival than either risk factor alone.

\section{Changes in Alignment With Time}

We found a tendency for knees that underwent revision to have drifted back into varus in the period between implantation and revision. While surgeons attempt to correct TKA alignment, and in most cases are successful (Figs. 6B and 7A), patients nonetheless continue to place high demands on their implants. For example, high adduction moments occur even during normal gait as the ground reaction force passes medial to the center of the knee [16]. Previous nonsurgical and surgical interventions have targeted the reduction of the adduction moment to slow progression of osteoarthritis [12, 16, 25]. However, despite restoration of static knee alignment, the adduction moment remains high after surgery [20], just as in a healthy native human knee. While we found a predisposition toward recurrence of the preoperative varus deformity, we did not find increased medial polyethylene damage. Therefore, the biomechanical consequences of the change in alignment from postoperatively to prerevision may be better reflected by collapse of underlying cancellous bone, the weak link in the load transfer pathway from the articular surface to the tibial cortex, than by increased polyethylene damage. This can be seen in the greater tendency for a drift back to varus in TKAs that experienced loosening compared with those revised for other reasons (Fig. 7B).

\section{Conclusions}

We found that damage was higher in TKAs with postoperative varus alignment. While understanding there may be other operative considerations, such as soft tissue balancing, our findings support restoring limb alignment as a way of reducing implant polyethylene damage and the subsequent risk of osteolysis caused by biological reaction to the released particulate wear debris. Like most studies of alignment after TKA, our study was focused on the static, coronal plane alignment and did not evaluate sagittal plane or rotational alignment, the combined effects of coronal, sagittal, and rotational alignment, or the dynamic effect of gait and other activities on alignment. We have a prospective study underway to examine the relationship between three-dimensional static alignment and dynamic function in patients who have had TKA. 
Acknowledgments We thank Elexis Baral BS (Department of Biomechanics, Hospital for Special Surgery, New York, NY) for assistance with implant grading.

\section{References}

1. Altman D. Practical Statistics for Medical Research. London, UK: Chapman and Hall; 1991.

2. Anderson JA, Baldini A, MacDonald JH, Tomek I, Pellicci PM, Sculco TP. Constrained condylar knee without stem extensions for difficult primary total knee arthroplasty. J Knee Surg. 2007;20:195-198.

3. Andrews M, Noyes FR, Hewett TE, Andriacchi TP. Lower limb alignment and foot angle are related to stance phase knee adduction in normal subjects: a critical analysis of the reliability of gait analysis data. J Orthop Res. 1996;14:289-295.

4. Andriacchi TP. Biomechanics and gait analysis in total knee replacement. Orthop Rev. 1988;17:470-473.

5. Berend ME, Ritter MA, Meding JB, Faris PM, Keating EM, Redelman R, Faris GW, Davis KE. Tibial component failure mechanisms in total knee arthroplasty. Clin Orthop Relat Res. 2004;428:26-34.

6. Bonner TJ, Eardley WG, Patterson P, Gregg PJ. The effect of post-operative mechanical axis alignment on the survival of primary total knee replacements after a follow-up of 15 years. $J$ Bone Joint Surg Br. 2011;93:1217-1222.

7. Centers for Disease Control and Prevention, National Center for Health Statistics. National Hospital Discharge Survey. Available at: https://www.cdc.gov/nchs/nhds/. Accessed May 31, 2017.

8. Collier MB, Engh CA Jr, McAuley JP, Engh GA. Factors associated with the loss of thickness of polyethylene tibial bearings after knee arthroplasty. J Bone Joint Surg Am. 2007;89:13061314.

9. D'Lima DD, Chen PC, Colwell CW. Polyethylene contact stresses, articular congruity, and knee alignment. Clin Orthop Relat Res. 2001;392:232-238.

10. D'Lima DD, Hermida JC, Chen PC, Colwell CW Jr. Polyethylene wear and variations in knee kinematics. Clin Orthop Relat Res. 2001;392:124-130.

11. Donaldson WF 3rd, Sculco TP, Insall JN, Ranawat CS. Total condylar III knee prosthesis: long-term follow-up study. Clin Orthop Relat Res. 1988;226:21-28.

12. Erhart JC, Mündermann A, Elspas B, Giori NJ, Andriacchi TP. A variable-stiffness shoe lowers the knee adduction moment in subjects with symptoms of medial compartment knee osteoarthritis. $J$ Biomech. 2008;41:2720-2725.

13. Fang DM, Ritter MA, Davis KE. Coronal alignment in total knee arthroplasty: just how important is it? J Arthroplasty. 2009;24(6 suppl):39-43.

14. Faris PM, Ritter MA, Davis KE, Priscu HM. Ten-year outcome comparison of the Anatomical Graduated Component and Vanguard total knee arthroplasty systems. J Arthroplasty. 2015;30:17331735.

15. Hood RW, Wright TM, Burstein AH. Retrieval analysis of total knee prostheses: a method and its application to 48 total condylar prostheses. J Biomed Mater Res. 1983;17:829-842.

16. Johnson F, Leitl S, Waugh W. The distribution of load across the knee: a comparison of static and dynamic measurements. $J$ Bone Joint Surg Br. 1980;62:346-349.
17. Longstaff LM, Sloan K, Stamp N, Scaddan M, Beaver R. Good alignment after total knee arthroplasty leads to faster rehabilitation and better function. $J$ Arthroplasty. 2009;24:570-578.

18. Meneghini RM, Mont MA, Backstein DB, Bourne RB, Dennis DA, Scuderi GR. Development of a modern Knee Society radiographic evaluation system and methodology for total knee arthroplasty. J Arthroplasty. 2015;30:2311-2314.

19. Morgan SS, Bonshahi A, Pradhan N, Gregory A, Gambhir A, Porter ML. The influence of postoperative coronal alignment on revision surgery in total knee arthroplasty. Int Orthop. 2008;32:639-642.

20. Orishimo KF, Kremenic IJ, Deshmukh AJ, Nicholas SJ, Rodriguez JA. Does total knee arthroplasty change frontal plane knee biomechanics during gait? Clin Orthop Relat Res. 2012;470:1171-1176.

21. Padgett DE, Cottrell J, Kelly N, Gelber J, Farrell C, Wright TM. Retrieval analysis of nonmodular constrained tibial inserts after primary total knee replacement. Orthop Clin North Am. 2012;43:e39e43.

22. Pang HN, Jamieson P, Teeter MG, McCalden RW, Naudie DD, MacDonald SJ. Retrieval analysis of posterior stabilized polyethylene tibial inserts and its clinical relevance. J Arthroplasty. 2014;29:365-368.

23. Parratte S, Pagnano MW, Trousdale RT, Berry DJ. Effect of postoperative mechanical axis alignment on the fifteen-year survival of modern, cemented total knee replacements. J Bone Joint Surg Am. 2010;92:2143-2149.

24. Petersen TL, Engh GA. Radiographic assessment of knee alignment after total knee arthroplasty. J Arthroplasty. 1988;3:67-72.

25. Prodromos CC, Andriacchi TP, Galante JO. A relationship between gait and clinical changes following high tibial osteotomy. J Bone Joint Surg Am. 1985;67:1188-1194.

26. Ritter MA. The Anatomical Graduated Component total knee replacement: a long-term evaluation with 20-year survival analysis. J Bone Joint Surg Br. 2009;91:745-749.

27. Ritter MA, Davis KE, Meding JB, Pierson JL, Berend ME, Malinzak RA. The effect of alignment and BMI on failure of total knee replacement. J Bone Joint Surg Am. 2011;93:1588-1596.

28. Schroer WC, Berend KR, Lombardi AV, Barnes CL, Bolognesi MP, Berend ME, Ritter MA, Nunley RM. Why are total knees failing today? Etiology of total knee revision in 2010 and 2011. $J$ Arthroplasty. 2013;28(8 suppl):116-119.

29. Sheehy L, Felson D, Zhang Y, Niu J, Lam YM, Segal N, Lynch J, Cooke TD. Does measurement of the anatomic axis consistently predict hip-knee-ankle angle (HKA) for knee alignment studies in osteoarthritis? Analysis of long limb radiographs from the multicenter osteoarthritis (MOST) study. Osteoarthritis Cartilage. 2011;19:58-64.

30. Srivastava A, Lee GY, Steklov N, Colwell CW Jr, Ezzet KA, D'Lima DD. Effect of tibial component varus on wear in total knee arthroplasty. Knee. 2012;19:560-563.

31. Vandekerckhove PT, Matlovich N, Teeter MG, MacDonald SJ, Howard JL, Lanting BA. The relationship between constitutional alignment and varus osteoarthritis of the knee. Knee Surg Sports Traumatol Arthrosc. 2016 Feb 1. [Epub ahead of print]

32. Wasielewski RC, Galante JO, Leighty RM, Natarajan RN, Rosenberg AG. Wear patterns on retrieved polyethylene tibial inserts and their relationship to technical considerations during total knee arthroplasty. Clin Orthop Relat Res. 1994;299:31-43.

33. Wright TM, Bartel DL. The problem of surface damage in polyethylene total knee components. Clin Orthop Relat Res. 1986;205:67-74. 